\title{
Epidemiological modelling for tropical disease control
}

\author{
J H F REMME
}

\section{Introduction}

The epidemiology of tropical diseases depends on the dynamic interaction of a multitude of biological, ecological, demographic and behavioural factors and processes, many of which cannot be measured directly. This makes it difficult to arrive at a comprehensive quantitative understanding of the epidemiology and control of tropical diseases without the aid of special epidemiometric tools. Epidemiological modelling can be helpful in this respect by providing a framework for the quantitative description of the dynamics of transmission, infection, disease and the impact of control.

Advances in model development and application have been modest for most tropical diseases and the practical value of models has often been questioned. In this paper I will briefly review a few examples of modelling in tropical diseases, with special reference to the case of onchocerciasis, and then identify what I consider to be the potential advantages and disadvantages of epidemiological modelling for tropical disease control. I will try to argue that epidemiological modelling can have great operational value if a number of conditions are met.

\section{Previous modelling in tropical diseases}

A good epidemiologial model is a simplication of reality which is nevertheless elaborate enough to give a realistic description of the main processes under study. The difficulty in epidemiological modelling is to strike a proper balance between simplification and realism. In the early days of modelling the emphasis was on simplification, but more recently the balance has started to swing back in favour of realism.

The first generation of models aimed at giving a simple and elegant description of the transmission and natural history of disease. These models were purely mathematical models which had to be simple to keep them mathematically tractable. However, their application involved the study of very complex issues such as the definition of breakpoint levels for the prevalence of infection or the vector density below which transmission cannot maintain itself and the infection will die out naturally. ${ }^{1}$ The often bitter experiences of eradication programmes and the results of detailed analysis of epidemiological data has shown that these early models were just too simple for these types of application. The subsequent generations of mathematical models have tried to explain 
why this was so by demonstrating the importance for transmission of such factors as density dependent regulation, heterogeneity in exposure and susceptibility to infection, and nonuniform impact of interventions. ${ }^{2,3}$ These mathematical models have been quite useful by clarifying concepts and improving the understanding of the transmission process.

The computer revolution has allowed a recent shift from purely mathematical models to computer simulation models which are less restricted by mathematical limitations and can better fulfil the requirement for realism. As a result, modelling has become more accepted among public health specialists and there are now several examples of the successful application of epidemiological simulation models for the prediction of epidemiological trends and for the planning and evaluation of tropical disease control programmes.

\section{THE EXAMPLE OF MALARIA}

The first model for malaria was a simple mathematical model developed during the first decade of this century by Sir Ronald Ross and highlighted the role of the vector in the transmission process. It indicated that not all vectors needed to be eliminated to ensure the interruption of transmission but that it was sufficient to reduce the vector density below a certain breakpoint level. Following this early start, further modelling development was slow and had to await the work of MacDonald in the 1950s and the major attempt of modelling malaria transmission as part of the WHO research project on the epidemiology and control of malaria in the Sudan savanna in West Africa, the so-called Garki project. The Garki model, in which the concept of immunity had been introduced, was quantified and tested using the parasitological and entomological data from the Garki project and proved to be a major improvement compared to previous malaria models. ${ }^{4}$ Its major drawback was that it was only a transmission model and did not address the question of morbidity and its control. In a review of their work, the investigators concluded that the Garki model has had important educational value even though it has not been used directly for planning of control. ${ }^{5}$

\section{THE EXAMPLE OF LEPROSY}

Modelling has played an important role in leprosy research because of the work by Lechat, who developed an epidemiometric model which used computer simulation. ${ }^{6,7}$ The emphasis in this work was on the relative comparison of alternative control options by simulating their long-term impact, and it has helped considerably to clarify the thinking about leprosy control. Further information about this model and its application are given in another article in this volume. ${ }^{8}$

\section{THE EXAMPLE OF LYMPHATIC FILARIASIS}

Since the 1960s, so-called catalytic models have been used extensively in the analysis of age-specific data on microfilaraemia. This work has resulted in estimates of the duration of microfilaraemia and of the force of infection. Comparison of the estimated force of infection and entomological data highlighted the inefficiency of transmission in lymphatic 
filariasis and indicated that only a very small proportion of inoculated larvae managed to develop into adult worms. Catalytic models have also been used to support alternative hypotheses on the natural history of disease which challenge the generally accepted theory and the role of immunological factors. ${ }^{22}$

Recently a new TDR initiative was launched for the accelerated development of epidemiological modelling in lymphatic filariasis for the purpose of steering research and to help the design of more effective control strategies. ${ }^{9}$ This initiative was partly a result of the success with epidemiological modelling for another filarial disease, onchocerciasis.

\section{THE EXAMPLE OF ONCHOCERCIASIS}

The Onchocerciasis Control Programme in West Africa (OCP), which is one of the largest tropical disease control programmes in the world, started its operations in 1975. The strategy of the Programme was to interrupt transmission by vector control for a period of 20 years, a period which was supposed to exceed the lifespan of the parasite even though the actual lifespan was not known. Epidemiological modelling was not used in the planning phase of the project and only af ter several years of control did it become obvious that there was a definite role for modelling in the OCP. After 8 years of control there was great uncertainty about the epidemiological impact of the control operations. The entomological evaluation results suggested that transmission had been interrupted but the epidemiological evaluation showed only a little decline in the prevalence of infection. This uncertainty about the impact of control, together with the lack of reliable predictions of future epidemiological trends and funding requirements, caused considerable nervousness in the donor community and it became urgent to resolve this issue. As a first step a simple force-of-infection model was developed to help interpret the extensive epidemiological data collected. ${ }^{10} \mathrm{~W}$ ith the new analytical approach it could be concluded from the epidemiological data that vector control had been successful in interrupting transmission in $90 \%$ of the OCP area and that the parasite reservoir was dying out rapidly. A first estimate of the average lifespan was arrived at and it was concluded that the required period of vector control was considerably less than 20 years. Subsequently, a more sophisticated computer simulation model was developed for the detailed prediction of future epidemiological trends under vector control and of the remaining vector control period required. The predictions of this model have been useful in securing funds for the third financial phase of the $\mathrm{OCP}^{11}$ and 5 years later it could be shown that the predictions were correct. ${ }^{12,13}$

In 1987 there was another development which prompted further epidemiological modelling. A new drug, Ivermectin, had successfully passed the clinical trials and become available for the treatment of onchocerciasis. Ivermectin is a microfilaricide which does not affect the adult worm but only kills its off spring, the microfilariae. This development required a review of the control strategy and a decision whether the future control strategy should be based on chemotherapy, vector control or a combination of the two. To help resolve this question, ONCHOSIM, a sophisticated computer simulation model for the transmission and control of onchocerciasis, was developed. ${ }^{14}$ This model has been used extensively in the OCP, not only for the comparison of the alternative control strategies but as a powerful epidemiological tool in day-to-day operational planning and evaluation of onchocerciasis control. 


\section{Advantages and disadvantages of epidemiological modelling}

Based on the rather unique experience with the operational application of modelling in the OCP I have listed below what I consider to be the potential advantages and disadvantages of epidemiological modelling for tropical disease control.

\section{ADVANTAGES}

\section{Comprehensive quantitative approach to epidemiology and control}

Because of the complexity of tropical diseases, epidemiological research is often designed to study a well-defined part of the overall problem in order to keep the study manageable and to obtain clear and unambiguous results. The disadvantage of this approach is that epidemiological research tends to be fragmented and not directly designed to address questions of control. Modelling forces a comprehensive approach to the main factors which determine the epidemiology and control of a disease, and to their interaction, and facilitates epidemiological reasoning focused on disease control.

\section{Integrated analysis and synthesis of knowledge and information}

It is of ten believed that modelling can only be meaningful for diseases for which the natural history is fully understood and can be quantified in detail. However, this belief is completely mistaken and the lack of quantitative information on important factors in the transmission and disease process may actually be a good reason for embarking on modelling. Different sets of information can, when analysed integrally using an epidemiological model, provide an estimate for important parameters which can otherwise not be quantified. An example in the OCP was the integrated analysis of the results of routine epidemiological evaluation data on infection levels, results on the viability of adult worms removed surgically from patients in a few selected villages, and entomological information on transmission levels. This analysis allowed a very satisfactory estimation of the parasite lifespan, an unknown but crucial factor which determines the required duration of the vector control programme. ${ }^{12,15}$

\section{Identification of research priorities and planning of research}

During the design and quantification of a model it soon becomes evident what the relative importance is of the different factors which determine the transmission and disease process, and what the most important information is which is lacking and requires (field) research. During the development of ONCHOSIM it became very clear that the relationship between vector infectivity and intensity of infection of the human host was extremely important for the dynamics of infection and special field experiments were undertaken to quantify this relationship. Without the modelling work this relationship would probably never have been studied in such detail. The relationship proved to be particularly important for predicting the impact of Ivermectin based control ${ }^{16}$ and it is currently believed that the observed difference in effectiveness of Ivermectin treatment for transmission control in the West African savanna and in Central America is due to the fact that the above relationship is very different for the vector species from these two 
areas. In West Africa, the vector is already highly efficient when the microfilarial loads of the human hosts are still low, such as after Ivermectin treatment, while the Central American vector hardly transmits at low microfilarial loads.

\section{Prospective evaluation of control strategies}

Prospective evaluation is usually seen as the most valuable application of modelling. It involves the prediction of the long-term impact of alternative control strategies and the prospective comparison of their (cost-)effectiveness. ${ }^{17}$ In some situations it may be sufficient to compare the relative effectiveness of alternative control strategies without requiring great accuracy in the predictions of the epidemiological trends over time. However, such accuracy becomes much more important when issues like long-term planning and cost considerations are considered. The most important OCP example of prospective evaluation was the comparison between the predicted long-term impact of chemotherapy and larviciding. The conclusion was that chemotherapy is useful for morbidity control if it can be sustained over a long period but that only larviciding for transmission control can achieve definite control within a time limited period. Vector control is therefore still the basis of the control strategy in the OCP. ${ }^{13,18}$

\section{Appreciation of uncertainty through sensitivity analysis}

A problem with detailed epidemiological models is that they involve many parameters which cannot be measured accurately but which do affect the model predictions. However, with modelling the uncertainty due to this can be studied in so-called sensitivity analyses. A practical example in the OCP was the sensitivity analysis of the risk of recrudescence if vector control would be stopped after a given number of years. This risk depends among others on the true biting rate and the variability in the parasite lifespan. The sensitivity analysis showed that the remaining uncertainty about the lifespan variability was not very important but that the risk of recrudescence was highly sensitive to variation in the biting rate, a conclusion which has had practical implications for the design of surveillance strategies. ${ }^{19,20}$

\section{Planning of control and evaluation activities}

Modelling can even be valuable for the planning of actual control and evaluation activities by indicating when and where it is most efficient to do what. An example in the OCP is the planning of surveillance activities after cessation of vector control. During the first 2 years this will involve only entomological surveillance for residual transmission at selected high-risk locations (the model was also used to predict which transmission levels would be acceptable and which not) but after 2 years this will be replaced by epidemiological surveillance for incidence of infection. ${ }^{20}$

\section{Prediction of epidemiological trends and funding requirements}

As mentioned above the prediction of trends is among the most important applications of epidemiological modelling. It involves the prediction of epidemiological changes during 
the control period but also of trends without control for the purpose of comparison and estimation of the benefit of control. These type of predictions are not only important for planning purposes but equally valuable for informing funding agencies and securing longterm funding. An important example in the OCP was the prediction of the trends in infection levels under vector control and the conclusion that 14 years of successful vector control were required. ${ }^{11,21}$ This conclusion was accepted by the donor community which made the required long-term funding commitment to the OCP.

\section{Evaluation of impact of control}

The predicted trends give a reference for the analysis of evaluation data on the impact of control. In the OCP this is the most common and routine application of modelling. An example is the epidemiological evaluation of the impact of vector control on transmission which was based on the comparison of observed trends in prevalence and intensity of skin microfilariae in the population of 187 indicator villages, ${ }^{5}$ which are surveyed at intervals of 3 years, with predicted epidemiological trends assuming complete interruption of transmission. ${ }^{13}$

\section{Continuous review}

The operational application of modelling involves continuous review of current epidemiological understanding through continuous testing of model predictions and analysis of evaluation and field research data. This process results in feedback and regular updating of the model to incorporate the new information and in improved understanding of the quantitative aspects of transmission and disease process.

\section{Interdisciplinary communication and collaboration in research and control}

Epidemiological modelling can be a great tool for improving communication between scientists of different disciplines involved in the epidemiology and control of tropical diseases. Model development in the OCP has brought together epidemiologists, entomologists, biostatisticians, parasitologists, ophthalmologists, managers, immunologists and behavioural scientists. There have been special multidisciplinary meetings for model development, and there are always extensive sessions during OCP research meetings to discuss simulation results and to review proposed model updates and planned applications.

\section{DISADVANT AGES}

\section{Oversimplification}

Oversimplification is the classical problem of modelling ${ }^{23}$ and, as mentioned before, the difficulty in modelling is to strike a proper balance between simplification and realism. Excessive simplification can be avoided by discussing the model assumptions with specialists in the various disciplines concerned and through detailed testing of the model against field data. 


\section{Overdose of mathematics and magic}

A classical problem with mathematical models is that an advanced mathematical description of an epidemiological problem is of limited use when attempting to communicate with public health specialists. The potential clients may be scared off by the formulas and not accept the conclusion of a model which they do not understand. This may also be a problem with simulation models, but less so if the model structure can be explained in a clear flow diagram which is epidemiologically meaningful.

\section{Uncritical interpretation}

A completely opposite problem occurs when those responsible for disease control operations start believing everything a model predicts. This may happen when some model applications have shown to be successful, as in the OCP example, and disease controllers lose their critical attitude to the model. It is the responsibility of the modeller to highlight the limitations of the model and to stress the need for continuous testing of assumptions and predictions.

\section{Cumbersome}

Serious epidemiological modelling involves a lot of work. This is particularly so for computer simulation models which require extensive computer programming, testing, debugging, quantification etc. It also requires specialized skills in mathematics and computer science, and scientists with experience in the application of modelling.

\section{High risk research}

It is always possible to make an epidemiological model for a disease, and it is very likely that a serious attempt at modelling will benefit the planning of epidemiological research. However, it is much less certain whether model development and testing will result in a model which is sufficiently valid and reliable to be directly useful for tropical disease control. Much will depend on the availability of epidemiological data on which the model can be tested.

\section{Conclusion}

Based on my experiences I would conclude that epidemiological modelling can be very valuable for tropical disease research and control if the following conditions are met. In the first place there should be a clear practical need and interest for epidemiological modelling among those involved in research and control of the disease. Secondly, model development, testing and application should be a truly multidisciplinary effort closely associated with control and field research activities. Thirdly, the structure of the model should be transparent and meaningful for all collaborating scientists. The actual transmission and disease process should therefore be modelled as closely as possible, pref erably by computer simulation. Fourthly, it is very important that modelling is seen as a continuing process and that a model evolves continuously through testing, application 
in analysis and updating when required. This implies that the initial model design should be flexible enough to allow future updating. Finally, those involved in field research and control should have direct access to the model and know how to use it as a practical epidemiological tool.

\section{References}

1 Bailey NTJ. The mathematical theory of infectious diseases and its applications. Griffin. London, 1975.

2 Anderson RM. Epidemiological models and predictions. Trop Geo Med, 1988; 40: S30-S39.

3 Dietz K. Density dependence in parasite transmission dynamics. Parasit Today, 1988; 4: 91-7.

4 Molineaux, L, Grammiccia G. The Garki Project. World Health Organization, Geneva, 1980.

5 Molineaux L. The pros and cons of modelling malaria transmission. Trans Royal Soc Trop Med Hyg, 1985; 79: $743-7$.

${ }^{6}$ Lechat MF, Misson JY, Vellut CM, Misson CB, Bouckaert A. Un modèle épidémiométrique de la lèpre. Bull WHO, 1974; 51: 361-73.

7 Lechat MF, Misson JY, Lambert A, Bouckaert A, Vanderveken M, Vellut CM. Simulation of vaccination and resistance in leprosy using an epidemiometric model. Int $J$ Lep, 1985; 53: 461-7.

8 Lechat MF. Epidemiometric modelling in leprosy based on Indian data. Lepr Rev, 1992; 63: Suppl. 31s-39s.

9 WHO. Informal consultation on epidemiological modelling for research and control of lymphatic filariasis. Mimeograghed document no. TDR/FIL/EPI-MOD/90.3. World Health Organization. Geneva, 1990.

10 Remme J, Ba O, Dadzie KY, Karam M. A force-of-infection model for onchocerciasis and its applications in the epidemiological evaluation of the Onchocerciasis Control Programme in the Volta River Basin area. Bull WHO, 1986; 64: 667-81.

11 WHO. Plan of operations for the third financial phase of the Onchocerciasis Control Programme in the Volta River Basin area (1986-1991). Mimeographed document no. OCP/85.2. World Health Organization. Geneva, 1985.

12 Remme J, De Sole G, Dadzie KY, Alley ES, Baker RHA, Habbema JDF, Plaisier AP, van Oortmarssen GJ, Samba EM. Large scale use of Ivermectin and its epidemiological consequences. Acta Leidensia, 1990a; 59: 177-92.

13 Remme J, De Sole G, van Oortmarssen GJ. The predicted and observed decline in the prevalence and intensity of onchocerciasis inf ection during 14 years of successf ul vector control. Bull WHO, 1990b; 68: 33139.

14 Plaisier AP, van Oortmarssen GJ, Habbema JDF, Remme J, Alley ES. ONCHOSIM, a simulation model for the transmission and control of onchocerciasis. Comp Meth Prog Biomed, 1990; 31: 43-56.

15 Plasier AP, van Oortmarssen GJ, Remme J, Habbema JDF. The reproductive lif espan of Onchocerca volvulus in West African savanna. Acta Tropica, 1991a; 48: 271-84.

16 WHO. Report of the Internal Research meeting of the Onchocerciasis Control Programme in West Africa. Mimeographed document no. OCP/EAC10/INF-DOC1. World Health Organization. Geneva, 1989b.

17 Habbema JDF, Plaisier AP, van Oortmarssen GJ, Remme J, Alley ES. Prospective evaluation of onchocerciasis control strategies. Acta Leidensia, 1990; 59: 387-98.

18 WHO. The prospective evaluation of onchocerciasis control in the Onchocerciasis Control Programme in West Africa; the application of a transmission model. Mimeographed document no. OCP/BIS/7.1/89/3. World Health Organization. Geneva, 1989a.

19 Plaisier AP, van Oortmarssen GJ, Remme J, Alley ES, Habbema JDF. The risk and dynamics of onchocerciasis recrudescence after cessation of vector control. Bull WHO, 1991b; 69: 169-78.

${ }^{20}$ WHO. Report of the Annual Internal Technical Review meeting of the Onchocerciasis Control Programme in West Africa. Mimeographed document no. OCP/EAC12.5. World Health Organization. Geneva, $1991 \mathrm{~b}$.

${ }^{21}$ WHO. Plan of operations for the fourth financial phase of the Onchocerciasis Control Programme. Mimeographed document no. OCP/EAC12.4. World Health Organization. Geneva 1991a.

22 Bundy DAT. The need for e pidemiological modelling in filariasis research and control. Working paper for third WHO Expert Committee on lymphatic filariasis. World Health Organization. Geneva 1991.

${ }^{23}$ Bradley DJ. Epidemiological models - theory and reality. In Anderson R M (ed.), Population dynamics of infectious diseases: theory and application. Chapman and Hall. London, 1982. 